\title{
Analytical model of the long-period forced longitude librations of Mercury
}

\author{
Marie Yseboodt ${ }^{1, *}$, Jean-Luc Margot ${ }^{2}$, Stanton J. Peale ${ }^{3}$ \\ ${ }^{1}$ Royal Observatory of Belgium, 3 Avenue Circulaire, 1180 Brussels, Belgium \\ 2 Earth and Space Sciences, University of California, Los Angeles, CA 90095, USA \\ ${ }^{3}$ Department of Physics, University of California, Santa Barbara, CA 93117, USA \\ * marie.yseboodt@oma.be
}

Icarus, Vol 207, doi: 10.1016/j.icarus.2009.12.020, 2010.

\begin{abstract}
The shaking of Mercury's orbit by the planets forces librations in longitude in addition to those at harmonics of the orbital period that have been used to detect Mercury's molten core. We extend the analytical formulation of Peale et al. (2009) in order to provide a convenient means of determining the amplitudes and phases of the forced librations without resorting to numerical calculations. We derive an explicit relation between the amplitude of each forced libration and the moment of inertia parameter $(B-A) / C_{m}$. Far from resonance with the free libration period, the libration amplitudes are directly proportional to $(B-A) / C_{m}$. Librations with periods close to the free libration period of $\sim 12$ years may have measurable ( $\sim \operatorname{arcsec}$ ) amplitudes. If the free libration period is sufficiently close to Jupiter's orbital period of 11.86 years, the amplitude of the forced libration at Jupiter's period could exceed the 35 arcsec amplitude of the 88-day forced libration. We also show that the planetary perturbations of the mean anomaly and the longitude of pericenter of Mercury's orbit completely determine the libration amplitudes.

While these signatures do not affect spin rate at a detectable level (as currently measured by Earth-based radar), they have a much larger impact on rotational phase (affecting imaging, altimetry, and gravity sensors). Therefore, it may be important to consider planetary perturbations when interpreting future spacecraft observations of the librations.
\end{abstract}

\section{Introduction}

Mercury is in a resonant state in which two revolution periods are equal to three rotation periods. This resonant state leads to librations in longitude whose periods are harmonics of the orbital period ( 88 days, 44d, 29d, etc.). These librations are due to the gravitational torque of the Sun on the permanent flattening of the planet. The amplitude of the 88-day libration is proportional to the moment of inertia ratio $(B-A) / C_{m}$ where $A<B$ are the moments of inertia of the planet and $C_{m}$ is the moment of inertia of the silicate shell about the spin axis.

Additionally to these main librations, Mercury also experiences planetary forced librations, due to the perturbations on the orbital motion of Mercury induced by the other planets. Therefore the associated periods are related to the orbital motion of the biggest planet (Jupiter) or to planets close to Mercury (Venus or the Earth). Peale et al $(2007,2009)$ give the amplitudes of these long-period forced librations using a numerical integration of the differential equations of motion while Dufey et al $(2008,2009)$ got the same amplitudes with an analytical study based on a Hamiltonian approach and Lie transforms. However for both of the above methods, a new calculation is necessary to determine the amplitudes for each specific value of $(B-A) / C_{m}$.

In this study, we give an analytical formulation for all the long-period forced librations of Mercury. This model is a generalization of the harmonic oscillator model given in Peale et al (2009) for the 11.86 year libration. The harmonic oscillator is damped by the core-mantle interactions and tidal dissipation and is periodically forced by the planetary perturbations of the orbit. 
Small planetary perturbations affecting the orbit of Mercury may produce non-negligible forced librations if their frequency is not too far from the free libration frequency. The planetary perturbations whose frequency is far away from the free libration frequency will force negligible librations. The main orbital elements leading to forced librations are the longitude of the pericenter of Mercury $\varpi$ and its mean anomaly $M$, whereas the perturbations of the semi-major axis and eccentricity yield negligible contributions.

Since Jupiter's orbital period of 11.86 years is within the $1 \sigma$ uncertainty of the free libration period of $\sim 12$ years consistent with the radar determination of $(B-A) / C_{m}$, a near resonance occurs with the free libration period for the 11.86 year orbit perturbation. So the amplitude of the forced libration at Jupiter's period could exceed the 35 arcsec amplitude of the 88-day forced libration (Peale et al. 2009, Dufey et al., 2008 and 2009). This happens whether the free libration is excited or not. The analytically determined amplitude of the 11.86 year forced libration as a function of proximity to resonance with the free libration period determined by the value of $(B-A) / C_{m}$ produces the numerically determined amplitude precisely on both sides of the resonance. Peale et al (2009) did not produce such a good fit because of a slight mismatch in the numerical and analytical libration angle definitions.

Since the free libration frequency is proportional to the square root of the moment of inertia ratio, our model provides a convenient means of determining the amplitudes and phases of the forced librations as a function of the moment of inertia parameter $(B-A) / C_{m}$ without resorting to numerical calculations. We derive an explicit relation between the amplitude of each forced libration and $(B-A) / C_{m}$, which amplitude is directly proportional to this parameter far from resonance with the free libration period.

The amplitude of the librations in longitude caused by the planetary perturbations of the orbit affect the spin rate at a level that may be too small to be detectable in the radar data. However, the angular orientation of Mercury about its spin axis may be monitored sufficiently precisely by ongoing and future spacecraft in orbit about Mercury to discern the amplitudes of at least the larger forced librations. Measurement of these amplitudes, especially that of the near resonant 11.86 year period, provide independent constraints on $(B-A) / C_{m}$, and will thereby reduce the uncertainty in this parameter.

We developed the model of a damped harmonic oscillator forced at the planetary frequencies and defined the libration angles more precisely in section 2 . The forcing due to the planets and the orbit perturbations is given in section 3. Then we discuss the libration amplitude (section 4) and phase (section 5). The link with the moments of inertia ratio is investigated in section 6 . Our model is synthesized in section 7 then fitted on the radar data in section 8 .

\section{Mercury's mantle as a damped harmonic oscillator forced at the planetary frequencies}

We consider Mercury as a two layer planet. At the considered timescale, it is commonly assumed that only the solid, upper part participates in the librations.

The motion of the mantle of Mercury due to the Sun, without any dissipation, is given by (e.g. Murray and Dermott, 1999):

$$
\psi_{m}^{\prime \prime}(t)=\frac{3(B-A) G M_{S}}{2 C_{m}} \frac{\sin \left(2 f(t)+2 \varpi(t)-2 \psi_{m}(t)\right)}{r(t)^{3}}
$$

where the angle $\psi_{m}(t)$ is the rotation angle of the mantle, it gives the orientation of the axis of minimum moment of inertia of the mantle relative to an inertial line joining the Sun to the intersection between the ecliptic and the orbital plane at J2000. $f(t)$ is the true anomaly, $M(t)$ is the mean anomaly, $a(t), e(t)$ and $\varpi(t)$ are the classical orbital elements, $r(t)$ is the distance between Mercury and the Sun, $A<B<C$ are the principal moments of inertia, $C_{m}$ the mantle moment of inertia. $M_{S}$ is the mass of the Sun, $G$ the gravitational constant. The angles are plotted in Fig. 1. The obliquity of Mercury is assumed to be 0 so that the equator and Mercury's orbit are equal.

Let's define the angle $\gamma_{m}(t)$ as:

$$
\psi_{m}(t)=\gamma_{m}(t)+1.5 M(t)+\varpi(t) .
$$

The main component of the rotation angle $\psi_{m}(t)$ is the sidereal rotation of Mercury while the angle $\gamma_{m}(t)$ is convenient to use in the following equations because it oscillates around 0 with a small amplitude and does not have any secular motion. The angle $\psi_{m}(t)$ references the rotation of Mercury with respect to an inertial line 

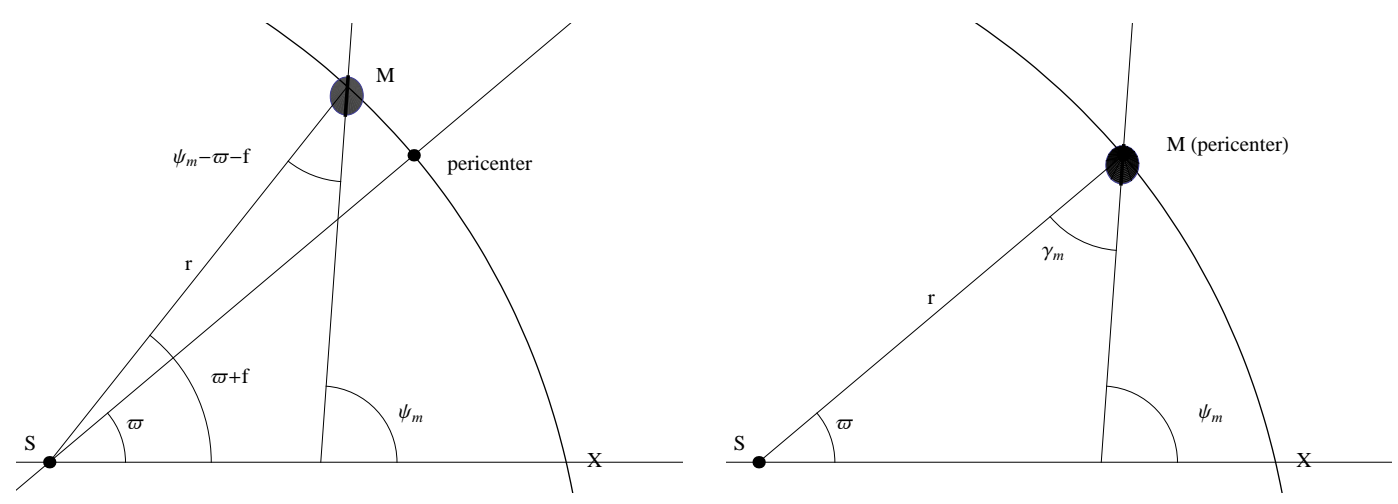

Figure 1: The angles used in our model. $\mathrm{M}$ is Mercury, SX is an inertial line between the Sun and the intersection between the ecliptic and the orbital plane at J2000. $\psi_{m}$ is the angle between SX and the axis of minimum moment of inertia. The right graph shows Mercury at pericenter $(M=f=0)$. At pericenter, the angle $\psi_{m}-\varpi$ is equal to $\gamma_{m}$.

while the angle $\gamma_{m}(t)$ combines both the rotation and the revolution. Because the definition of $\gamma_{m}(t)$ includes the mean anomaly (see Eq. 2), this angle does not have any simple geometrical interpretation, except at pericenter where $\psi_{m}=\gamma_{m}+\varpi$ (see the right part in Fig. 1). We can think of $\gamma_{m}(t)$ as a libration of the axis of minimum moment of inertia of Mercury about the Mercury-Sun line when Mercury is at perihelion (Fig. 1).

Then Eq. 1 becomes

$$
\gamma_{m}^{\prime \prime}(t)+1.5 M^{\prime \prime}(t)+\varpi^{\prime \prime}(t)=\frac{3(B-A) G M_{S}}{2 C_{m}} \frac{\sin \left(2 f(t)-3 M(t)-2 \gamma_{m}(t)\right)}{r(t)^{3}} .
$$

Replacing the distance between the Sun and Mercury $r(t)$ and also the $\cos f$ and $\sin f$ with series of eccentricity functions $G_{20 q}$ of Kaula (1966), we have

$$
\gamma_{m}^{\prime \prime}(t)+1.5 M^{\prime \prime}(t)+\varpi^{\prime \prime}(t)=-\sum_{q} \frac{3(B-A) G M_{S} G_{20 q}(e)}{2 C_{m} a(t)^{3}} \sin \left(2 \gamma_{m}(t)+(1-q) M(t)\right) .
$$

The free libration frequency $w_{0}$ can be easily obtained for $q=1$ if the variations in the orbital elements are neglected:

$$
w_{0}=n \sqrt{3(B-A) G_{201}(e) / C_{m}}
$$

where $n$ is the mean motion $n=\sqrt{G M_{S} / a^{3}}$ and $G_{201}(e)=7 e / 2-123 e^{3} / 16+O\left(e^{5}\right)$. The other $q$ values give the forced librations with periods equal to the harmonics of the orbital period.

Since we are looking at the long-period librations, the classical technique of averaging over one orbit period (88 days) is used and the short-period librations are neglected.

$$
\gamma_{m}^{\prime \prime}(t)+1.5 M^{\prime \prime}(t)+\varpi^{\prime \prime}(t) \approx-\frac{3(B-A) G M_{S} G_{201}(e)}{C_{m} a(t)^{3}} \gamma_{m}(t)
$$

The main perturbation of the time evolution of the angle $\gamma_{m}(t)$ after integration of the differential equation (6) is due to the $1.5 M^{\prime \prime}(t)+\varpi^{\prime \prime}(t)$ term in the left hand side. In the right hand side, the variations in the orbital elements change the value of $G_{201}$ through $e(t)$ in the numerator and also $a(t)^{3}$ in the denominator. Since these two quantities are multiplied by the small quantity $\gamma_{m}(t)$ (usually a few tens of arcseconds), their variations do not affect largely the evolution of the angle $\gamma_{m}(t)$ (second order perturbations).

We define the orbital variable $\lambda(t)$ as

$$
\lambda(t)=1.5 M(t)+\varpi(t) .
$$

If $w_{i}$ are the frequencies of the changing orbital motion of Mercury, the periodic part of $M(t)$ can be expressed as $\sum_{i} M_{i} \cos \left(w_{i} t+\phi_{M_{i}}\right)$ and the periodic part of $\varpi(t)$ as $\sum_{i} \varpi_{i} \cos \left(w_{i} t+\phi_{\varpi_{i}}\right)$. The new combination of orbital 
elements can be expressed as a sum of planetary frequencies

$$
\lambda(t)=\lambda_{0}+\lambda_{t} t+\sum_{i} \lambda_{i} \cos \left(w_{i} t+\phi_{\lambda_{i}}\right) .
$$

$\lambda_{0}$ and $\lambda_{t}$ are the initial value and secular increase respectively of the angle $\lambda(t)$ and are not used in the following development. The coefficients of the frequency development $\lambda_{i}$ and $\phi_{\lambda_{i}}$ are related to the corresponding development for $M(t)$ and $\varpi(t)$ by the following relations:

$$
\lambda_{i}=\sqrt{\frac{9}{4} M_{i}^{2}+\varpi_{i}^{2}+3 \varpi_{i} M_{i} \cos \left(\phi_{M_{i}}-\phi_{\varpi_{i}}\right)}
$$

and

$$
\tan \phi_{\lambda_{i}}=\frac{1.5 M_{i} \sin \phi_{M_{i}}+\varpi_{i} \sin \phi_{\varpi_{i}}}{1.5 M_{i} \cos \phi_{M_{i}}+\varpi_{i} \cos \phi_{\varpi_{i}}}
$$

All the amplitudes in this paper are chosen positive, the sign being included in the phase. The second derivative of the orbital variable $\lambda(t)$ is

$$
\lambda^{\prime \prime}(t)=1.5 M^{\prime \prime}(t)+\varpi^{\prime \prime}(t)=-\sum_{i} w_{i}^{2} \lambda_{i} \cos \left(w_{i} t+\phi_{\lambda_{i}}\right),
$$

$\lambda^{\prime \prime}(t)$ is the forcing of the system due to the planets. If we add the tidal and core-mantle dissipation (Peale et al, 2009), Eq. 6 becomes

$$
\gamma_{m}^{\prime \prime}(t)-\sum_{i} w_{i}^{2} \lambda_{i} \cos \left(w_{i} t+\phi_{\lambda_{i}}\right)=-\frac{3(B-A) G M_{S} G_{201}(e)}{C_{m} a^{3}} \gamma_{m}(t) \underbrace{-\left(\frac{F}{C_{m} n}+\frac{k}{C_{m}}\right) \gamma_{m}^{\prime}(t)-\frac{F D}{C_{m}}+\frac{k}{C_{m}} \gamma_{c}^{\prime}(t)}_{\text {dissipation }}
$$

where $\gamma_{c}(t)$ is the libration of the core, $k$ is a constant coupling the core and the mantle, $F$ is a constant depending on the mantle properties and shape and $D$ a function of the eccentricity.

The dissipation parameter is defined as $b=F /\left(C_{m} n\right)+k / C_{m}$.

If $\gamma_{c}^{\prime}(t)$ can be neglected, Eq. 12 is the equation of a damped harmonic oscillator forced at the frequencies $w_{i}$. The constant term $-F D / C_{m}$ from the tidal dissipation leads to a small shift in the center of libration (Peale et al 2007), which will be neglected.

The well-known solution of the damped harmonic oscillator forced sinusoidally at the $w_{i}$ frequency is

$$
\gamma_{m}(t)=\exp (-b t / 2) D_{f l}^{\prime} \cos \left(w_{0}^{\prime} t+\phi_{f l}\right)+\sum_{i} \gamma_{i} \cos \left(w_{i} t+\phi_{i}\right)
$$

where $w_{0}^{\prime}=\sqrt{w_{0}^{2}-b^{2} / 4}, \phi_{f l}$ and $D_{f l}^{\prime}$ are determined by the initial conditions. The first part of the solution of $\gamma_{m}(t)$ is the free libration while the second part is the response to the orbital forcing.

The phase of the planetary libration $\phi_{i}=\phi_{\lambda_{i}}+\phi_{i}^{R}$ is the sum of the planetary phase and the phase of the oscillation relative to the driving force where the phase $\phi_{i}^{R}$ is given by

$$
\begin{aligned}
\sin \phi_{i}^{R} & =-w_{i} b / \sqrt{\left(w_{0}^{2}-w_{i}^{2}\right)^{2}+w_{i}^{2} b^{2}} \\
\cos \phi_{i}^{R} & =\left(w_{0}^{2}-w_{i}^{2}\right) / \sqrt{\left(w_{0}^{2}-w_{i}^{2}\right)^{2}+w_{i}^{2} b^{2}}
\end{aligned}
$$

If the dissipation is neglected, the phase $\phi_{i}^{R}$ is 0 for the frequencies smaller than the free libration frequency and is $180^{\circ}$ for the frequencies above $w_{0}$.

The amplitude $\gamma_{i}$ due to the planetary perturbation of the orbit is

$$
\gamma_{i}=\lambda_{i} w_{i}^{2} / \sqrt{\left(w_{0}^{2}-w_{i}^{2}\right)^{2}+w_{i}^{2} b^{2}} .
$$

When $w_{0}<w_{i}$ and the frequency $w_{i}$ is far from the resonance frequency $w_{0}$, since $b$ is small with respect of $w_{i}$, the coefficient $\gamma_{i}$ (Eq. 15) is reduced to

$$
\gamma_{i} \approx \lambda_{i}\left(1+\frac{w_{0}^{2}}{w_{i}^{2}}\right) \approx \lambda_{i}\left(1+\frac{(B-A)}{C_{m}} \frac{3 n^{2} G_{201}(e)}{w_{i}^{2}}\right)
$$


When $w_{0}>w_{i}$ and if the frequency $w_{i}$ is far from the resonance frequency $w_{0}$, the coefficient $\gamma_{i}$ is $\gamma_{i} \approx$ $\frac{\lambda_{i}}{w_{0}^{4}}\left(w_{i}^{4}+w_{0}^{2} w_{i}^{2}\right)$.

However the $\gamma_{m}$ angle is difficult to interpret. The rotation variation angle $\psi_{m}(t)$ is more relevant in most cases. For example, the amplitudes of Table 1 in Peale et al (2009) are given for the angle $\psi_{m}(t)$. The link between the amplitude of these two angles $\psi_{i}$ and $\gamma_{i}$ is

$$
\psi_{i}=\sqrt{\gamma_{i}^{2}+\lambda_{i}^{2}+2 \gamma_{i} \lambda_{i} \cos \phi_{i}^{R}} .
$$

For $w_{0} \ll w_{i}$ and no dissipation, the amplitude $\psi_{i}$ becomes:

$$
\psi_{i} \approx \gamma_{i}-\lambda_{i} \approx \frac{\lambda_{i}}{w_{i}^{2}} \frac{(B-A)}{C_{m}} 3 n^{2} G_{201}(e) .
$$

For $w_{0} \gg w_{i}$, the amplitude $\psi_{i}$ is $\psi_{i}=\gamma_{i}+\lambda_{i}$.

The phase of $\psi_{m}(t)$ is given by:

$$
\tan \phi_{\psi_{i}}=\frac{\gamma_{i} \sin \left(\phi_{\lambda_{i}}+\phi_{i}^{R}\right)+\lambda_{i} \sin \phi_{\lambda_{i}}}{\gamma_{i} \cos \left(\phi_{\lambda_{i}}+\phi_{i}^{R}\right)+\lambda_{i} \cos \phi_{\lambda_{i}}} .
$$

Again, without dissipation, this angle is reduced to $\phi_{\psi_{i}} \approx \phi_{\lambda_{i}}+\phi_{i}^{R}$.

\section{The forcing due to the planets}

The amplitudes $\left(\varpi_{i}\right.$ and $\left.M_{i}\right)$ and the phases $\left(\phi_{\varpi_{i}}\right.$ and $\left.\phi_{M_{i}}\right)$ of the orbital elements can be found for each $w_{i}$ frequency on an interval close to the J2000 epoch. The dominant variations in the $\lambda$ variable are plotted in Fig. 2 and the numerical values are given in Table 1. The ephemeris used is the DE408 and the method used is a Fourier analysis. Using another ephemeris or another method will slightly change the forcing amplitudes but the corresponding changes for the libration amplitudes will also be very small (smaller than 0.1 as).

\begin{tabular}{|cc|cc|cc|cc|}
\hline $\begin{array}{c}\text { period } \\
2 \pi / w_{i}(\mathrm{y})\end{array}$ & $\begin{array}{c}\text { Forcing } \\
\text { argument }\end{array}$ & $\begin{array}{c}\varpi_{i} \\
\left(10^{-5} \mathrm{rad}\right)\end{array}$ & $\begin{array}{c}\phi_{\varpi_{i}} \\
(\mathrm{deg})\end{array}$ & $\begin{array}{c}M_{i} \\
\left(10^{-5} \mathrm{rad}\right)\end{array}$ & $\begin{array}{c}\phi_{M_{i}} \\
(\mathrm{deg})\end{array}$ & $\begin{array}{c}\lambda_{i} \\
(\mathrm{as})\end{array}$ & $\begin{array}{c}\phi_{\lambda_{i}} \\
(\mathrm{deg})\end{array}$ \\
\hline 5.663 & Venus $\left(2 \lambda_{M}-5 \lambda_{V}\right)$ & 1.64 & 271 & 5.19 & 88 & 12.7 & 87 \\
5.932 & Jupiter $\left(2 \lambda_{J}\right)$ & 3.51 & 183 & 3.73 & 4 & 4.31 & 4 \\
6.575 & Earth $\left(\lambda_{M}-4 \lambda_{E}\right)$ & 0.29 & 159 & 0.64 & 334 & 1.40 & 332 \\
11.864 & Jupiter $\left(\lambda_{J}\right)$ & 0.75 & 21 & 0.92 & 187 & 1.40 & 170 \\
14.727 & Saturn $\left(2 \lambda_{S}\right)$ & 0.42 & 215 & 0.45 & 35 & 0.52 & 35 \\
\hline 1.110 & $\left(\lambda_{M}-2 \lambda_{V}\right)$ & 2.16 & 56 & 3.90 & 235 & 7.59 & 235 \\
1.380 & $\left(\lambda_{M}-3 \lambda_{V}\right)$ & 1.20 & 52 & 0.93 & 230 & 0.40 & 222 \\
0.251 & & 0.41 & 351 & 0.53 & 171 & 0.79 & 172 \\
0.555 & $\left(2 \lambda_{M}-4 \lambda_{V}\right)$ & 0.40 & 199 & 0.59 & 18 & 1.01 & 17 \\
0.465 & & 0.37 & 218 & 0.53 & 38 & 0.88 & 38 \\
3.954 & $\left(3 \lambda_{J}\right)$ & 0.37 & 214 & 0.39 & 34 & 0.72 & 160 \\
0.615 & & 0.35 & 196 & 0.45 & 16 & 0.65 & 16 \\
0.292 & & 0.32 & 127 & 0.53 & 305 & 0.97 & 305 \\
0.241 & & 0.32 & 266 & 0.41 & 86 & 0.62 & 86 \\
\hline
\end{tabular}

Table 1: Planetary perturbations of Mercury's orbit and amplitudes of the $\lambda(t)=1.5 M(t)+\varpi(t)$ angle. The five waves that give the largest forced librations are given above the line, others perturbations are given below the line.

The annual perturbation due to Jupiter is $\lambda_{11.86 y}=1.4$ as (see Table 1). This is smaller than the semi-annual perturbation due to Jupiter whose period is 5.93 years. The phase of $\varpi_{i}$ is approximately opposite to the phase of $M_{i}$ for each frequency.

In Table 1, the correspondence between the frequencies and the forcing arguments (a combination of the longitudes of the planets) is only tentative and is not exact because the frequencies come from a numerical Fourier analysis and not from an analytical theory. Numerically, close frequencies in the orbital theory may be combined into a single perturbation.

Using the same ephemeris, the variations in the semi-major axis and in the eccentricity for the $w_{i}$ frequencies are smaller than 10 meters and $1.5 \times 10^{-6}$ respectively. This strengthens our simplifying hypotheses of neglecting the small variations in $a(t)$ and $e(t)$ in the harmonic oscillator model for the motion of the mantle. 


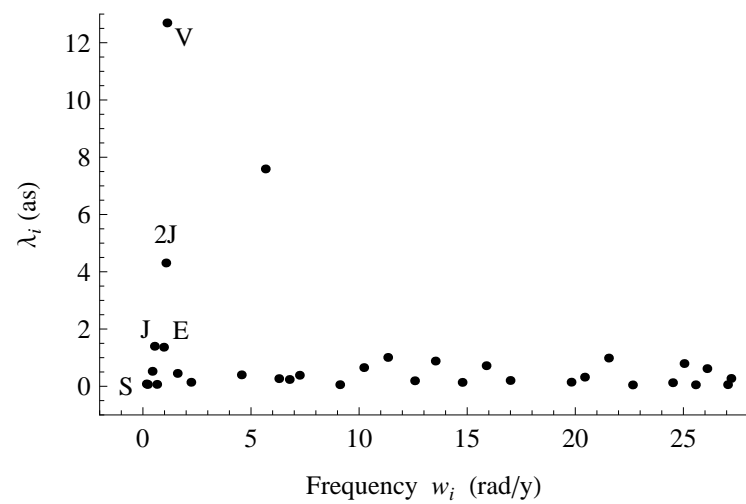

Figure 2: The 25 largest amplitudes of the orbital variable $\lambda_{i}$ and their frequencies $w_{i}$ around the J2000 epoch. The perturbations that mostly affect the libration in longitude are labeled with a letter corresponding to the perturbating planet (E for the Earth, S for Saturn, J for Jupiter and V for Venus).

\section{The libration amplitude}

In order to have a significant long-period forced libration at some orbital frequency $w_{i}$, two conditions must be fulfilled at the same time: the amplitude of orbital variable $\lambda_{i}$ at the orbital frequency $w_{i}$ must be large (see previous section and the amplitudes in Table 1), and the forcing frequency has to be close to the free libration frequency. In this section, we present the response to the driving perturbation and the corresponding libration amplitude.

The amplitude $\gamma_{i}$ is given by Eq. 15 and is proportional to the amplitude of the orbital variable $\lambda_{i}$. The factor is $w_{i}^{2} / \sqrt{\left(w_{0}^{2}-w_{i}^{2}\right)^{2}+w_{i}^{2} b^{2}}$. This factor is large if the forcing period is close to the free libration period.

If the $(B-A) / C_{m}$ ratio has the nominal value of $2.03 \times 10^{-4}$ as determined by Margot et al (2007), the free libration frequency $w_{0}$ value is $0.52 \mathrm{rad} / \mathrm{y}$ and its period is 12.07 years. For frequencies within $0.2 \mathrm{rad} / \mathrm{y}$ of this nominal resonant frequency, the orbit perturbation is multiplied by a factor larger than 1 .

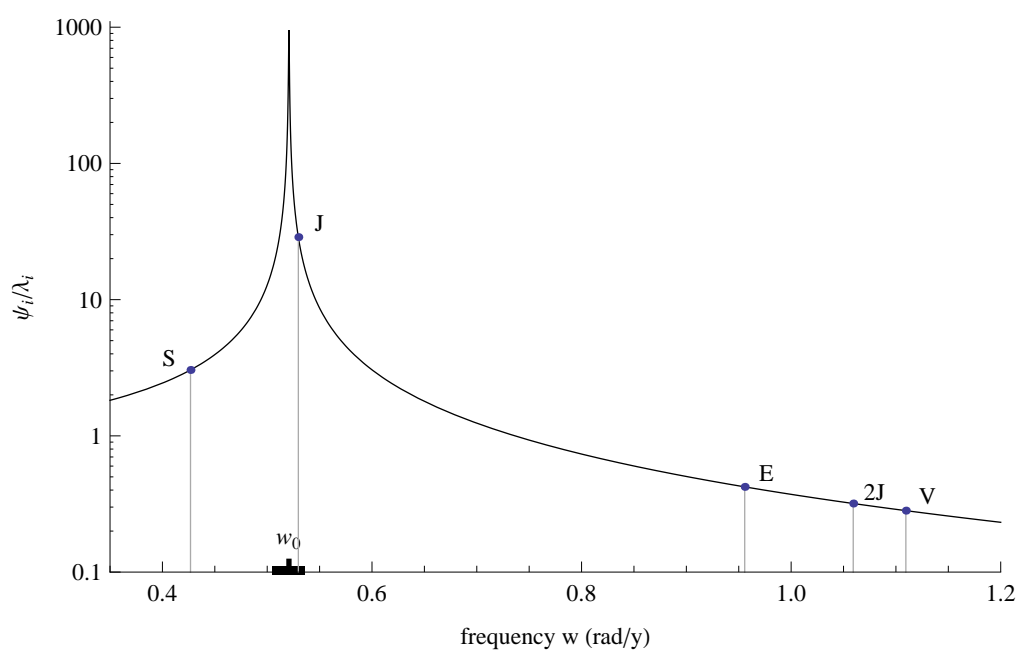

Figure 3: Rotation angle amplitude normalized by the amplitude of the planetary perturbation of the orbit $\lambda_{i}$ as a function of the frequency $w$. The quantity plotted is the ratio of $\psi_{i} / \lambda_{i}$ which is $\sqrt{1+\gamma_{i}^{2} / \lambda_{i}^{2}+2 \gamma_{i} / \lambda_{i} \cos \phi_{i}^{R}}$. The planetary frequencies $w_{i}$ are indicated with dots and are labeled. The nominal free libration period $w_{0}$ for the nominal value of $(B-A) / C_{m}$ from radar data and its uncertainty is also shown. The damping parameter $b$ is large $\left(5 \times 10^{-4} / \mathrm{y}\right)$.

Figure 3 shows the rotation angle amplitude normalized by the amplitude of the planetary perturbation of the orbit for a range of frequencies, including the planetary frequencies $w_{i}$ in Table 1 . The figure also shows the resonance with the free libration for periods close to 12 years. On the left part of the resonance corresponding 
to high periods, this ratio tends to 1 while on right hand side of the resonance, far from $w_{0}$, this ratio tends to 0 , meaning that any short-period perturbation of the orbital variable produces negligible libration for the angle $\psi_{m}$. These limits are independent of the damping. Far away from the resonance, the dissipation parameter $b$ can be neglected.

However between these two limits, there are a few orbital frequencies that leads to libration amplitude above 0.5 arcsecond (see the numerical values in Table 2). These five waves are Saturn (14.73y), Jupiter (11.86y and 5.93y), the Earth (6.57y) and Venus (5.66y). Their amplitudes are 1.6, 40, 1.4, 0.6 and 3.6as respectively. Depending on Mercury's moments of inertia, the amplitude of the $w_{11.86 y}$ libration due to Jupiter can be larger or smaller. The four other modes do not have very large amplitude because their period is not very close to the resonance, but their response is still non-negligible, and they need to be taken into account into future data analysis. Saturn $\left(w_{14.7 y}\right)$ perturbation is the only large wave on the left hand side of the resonance.

\begin{tabular}{|cc|c|c|c|c|}
\hline & $\begin{array}{c}\text { period } \\
2 \pi / w_{i}(\mathrm{y})\end{array}$ & $\begin{array}{c}w_{i}^{2} \lambda_{i} \\
\left(\mathrm{as} / \mathrm{y}^{2}\right)\end{array}$ & $\begin{array}{c}\psi_{i} \\
(\mathrm{as})\end{array}$ & $\begin{array}{c}\gamma_{i} \\
(\mathrm{as})\end{array}$ & $\begin{array}{c}\psi_{i} w_{i} \\
(\mathrm{as} / \mathrm{d})\end{array}$ \\
\hline Venus & 5.66 & 15.6 & 3.59 & 16.28 & 0.011 \\
Jupiter & 5.93 & 4.8 & 1.37 & 5.68 & 0.004 \\
Jupiter & 11.86 & 0.4 & 40.25 & 41.65 & 0.058 \\
Earth & 6.57 & 1.2 & 0.58 & 1.94 & 0.002 \\
Saturn & 14.72 & 0.1 & 1.6 & 1.07 & 0.002 \\
\hline & 1.11 & 243.3 & 0.06 & 7.66 & 0.0015 \\
& 0.55 & 129.3 & 0 & 1.01 & 0.0001 \\
& 0.29 & 457.3 & 0 & 0.99 & 0 \\
& 0.46 & 161.3 & 0 & 0.88 & 0 \\
0.25 & 497.7 & 0 & 0.79 & 0 \\
0.4 & 181.2 & 0 & 0.72 & 0 \\
& 0.62 & 68.1 & 0 & 0.65 & 0 \\
0.2 & 648.7 & 0 & 0.64 & 0 \\
0.24 & 420.2 & 0 & 0.62 & 0 \\
3.95 & 1.1 & 0.05 & 0.5 & 0.0002 \\
\hline
\end{tabular}

Table 2: The amplitudes of the largest long-period forced librations for the nominal value of the moments of inertia ratio. The five librations above 0.5 as are given above the horizontal line. The amplitudes are given for the rotation angle $\psi_{i}$ and the angle $\gamma_{i}$ (see the text for definition). The last column gives the amplitude of the libration rate.

Even if Fig. 3 shows a continuous curve, the librations cannot happen for any value of the frequency $w$, only the frequencies forcing the system (the planetary frequencies $w_{i}$ ) can be seen. These five periods are usually the only ones to be considered because their amplitude in the orbital theory is non negligible (see Table 1 and Fig. 2) and their period is sufficiently close enough to the free libration period. The orbital elements of Mercury have a lot of perturbations whose periods are shorter than two years. But they are far away from the resonance and the libration amplitude is much smaller than 1 as. For example, Venus induces a large variation in the $\lambda$ orbital variable with an amplitude of 7.6 as at the $1.11 \mathrm{y}$ period. Since this frequency is far away from $w_{0}$, this forcing frequency induces a libration with a negligible amplitude. However if the rotation measurements become more precise, the other frequencies should be taken into account by the same model.

The amplitude at the resonance will not be infinite because there is always some damping (see Eq. 15). The maximal amplitude for $\gamma(t)$ is $\lambda_{i} w_{0} / b$. The coefficient of $\lambda_{i}$ can be large as 1100 for $w_{0}=w_{i}=2 \pi / 11.86 \mathrm{y}$ and for a very high damping with a damping parameter $b$ of $5 \times 10^{-4} / \mathrm{y}$. For more reasonable damping, the damping parameter $b$ is about $10^{-5} / \mathrm{y}$ (the free libration is damped with a time scale of about $2 \times 10^{5}$ years, Peale 2005) and the maximal value of the amplitude is even larger. Therefore a small perturbation due to Jupiter of the orbital variable of Mercury with $\lambda_{11.86 y}=1.4$ as may induce a large effect in the libration angle. The parameters related to the dissipation have a large theoretical uncertainty, but this uncertainty affects very slightly the libration amplitudes. For example, if the dissipation parameter value is multiplied by a factor 10, the only amplitude that will decrease by more than 0.2 as is the 11.86 year libration if the free libration frequency is between 0.528 and $0.531 \mathrm{rad} / \mathrm{y}$ (corresponding to a period interval of 0.06 year).

All the plots in this paper have been computed with a large dissipation parameter $b$ of $5 \times 10^{-4} / \mathrm{y}$.

Because of the damping, the free libration period changes from $2 \pi / w_{0}$ to $2 \pi / w_{0}^{\prime}$. The difference is very small: 50 
seconds for a high damping, even less if the damping value is reasonable. The shift of the libration phase due to the dissipation $F D / C_{m}$ (see eq. 12 or Peale et al 2007) for a reasonable value $k_{2} / Q$ of 0.004 is about 1.5 as.

\section{The libration phase}

Figure 4 shows the phase shift relative to the driving force $\phi_{i}^{R}$ as a function of the frequencies $w$ (Eq. 14). This quantity has to be added to the orbital perturbation phase in order to get the libration phase.

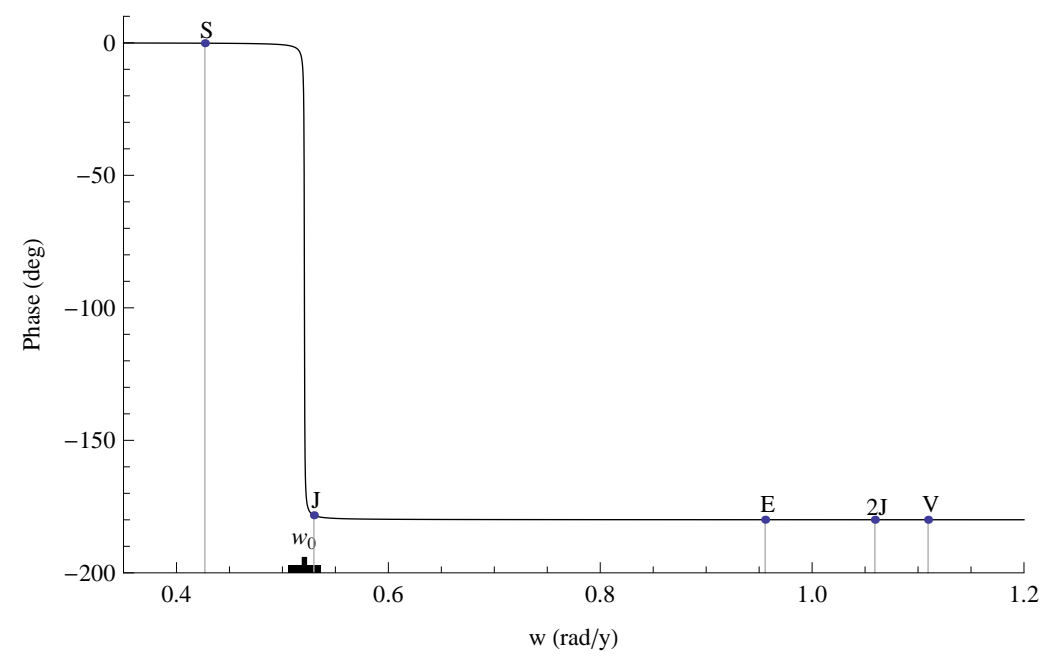

Figure 4: Phase shift relative to the driving force $\phi_{i}^{R}$ as a function of the frequency $w$.

Far from the resonance period, the phase shift is equal to 0 for frequencies $w_{i}$ smaller than $w_{0}$ or $180^{\circ}$ for frequencies larger than $w_{0}$. Close to the resonance with the $w_{0}$ frequency, the phase shift decreases rapidly from 0 to -180 degrees when frequency $w$ increases. The speed of decrease is a function of the dissipation parameter $b$. If the damping decreases from our large $b$ value and is closer to the expected damping, the transition from $0^{\circ}$ to $-180^{\circ}$ becomes even more abrupt. Very close to the resonance, a change in the dissipation will affect more the phase than the amplitude of the 11.86 year libration. For example, if $w_{0}$ is in the $[0.528,0.531] \mathrm{rad} / \mathrm{y}$ interval, the phase change will be larger than $2^{\circ}$ if the dissipation is multiplied by a factor 10 .

This opposite phase lag is important for the definition of $\psi_{i}$ (see Eq. 17) because it changes the sign of the $\lambda_{i}$ contribution. If the frequency $w_{i}$ is smaller than the free libration frequency $w_{0}$ (left side of the resonance) then $\psi_{i} \approx \gamma_{i}+\lambda_{i}$; while if the frequency is larger than $w_{0}$ (right side of the resonance) then $\psi_{i} \approx \gamma_{i}-\lambda_{i}$. On the left side of the resonance, when frequencies are decreasing, the $\gamma_{i}$ libration amplitude tends to the 0 value while on the right part of the resonance when frequencies are increasing, $\gamma_{i}$ tends to $\lambda_{i}$. Because of this $180^{\circ}$ phase shift, the behavior of the $\psi_{m}$ amplitude is the opposite: for small frequencies, it tends to $\lambda_{i}$ while for large frequencies, it tends to 0 .

\section{Link with the moment of inertia ratio}

The free libration frequency $w_{0}$ is proportional to the square root of the moment of inertia ratio (see Eq. 5). Therefore we can investigate the link between the forced libration amplitudes and the moment of inertia. It is worth to study this resonance in detail since the orbital period of Jupiter is in the $1 \sigma$ uncertainty interval for the free libration period based on $(B-A) / C_{m}$ ratio determined with the radar data.

If the $(B-A) / C_{m}$ ratio increases from the nominal value, the resonant frequency $w_{0}$ moves to the right in Fig. 3 and the $11.86 \mathrm{y}$ libration amplitude is larger since it is closer to the resonant frequency. Then after the passage through the resonance, its amplitude decreases.

The libration amplitude given by Eq. 15 is plotted in Fig. 5 as a function of the $(B-A) / C_{m}$ ratio for the different planetary frequencies $w_{i}$. The amplitude of the 11.86y libration is large because of the resonance effect with the free libration when the $(B-A) / C_{m}$ ratio is close to $2.1 \times 10^{-4}$.

Equation (18) shows that the amplitudes of the long-period forced libration for frequencies larger than $w_{0}$ are, 


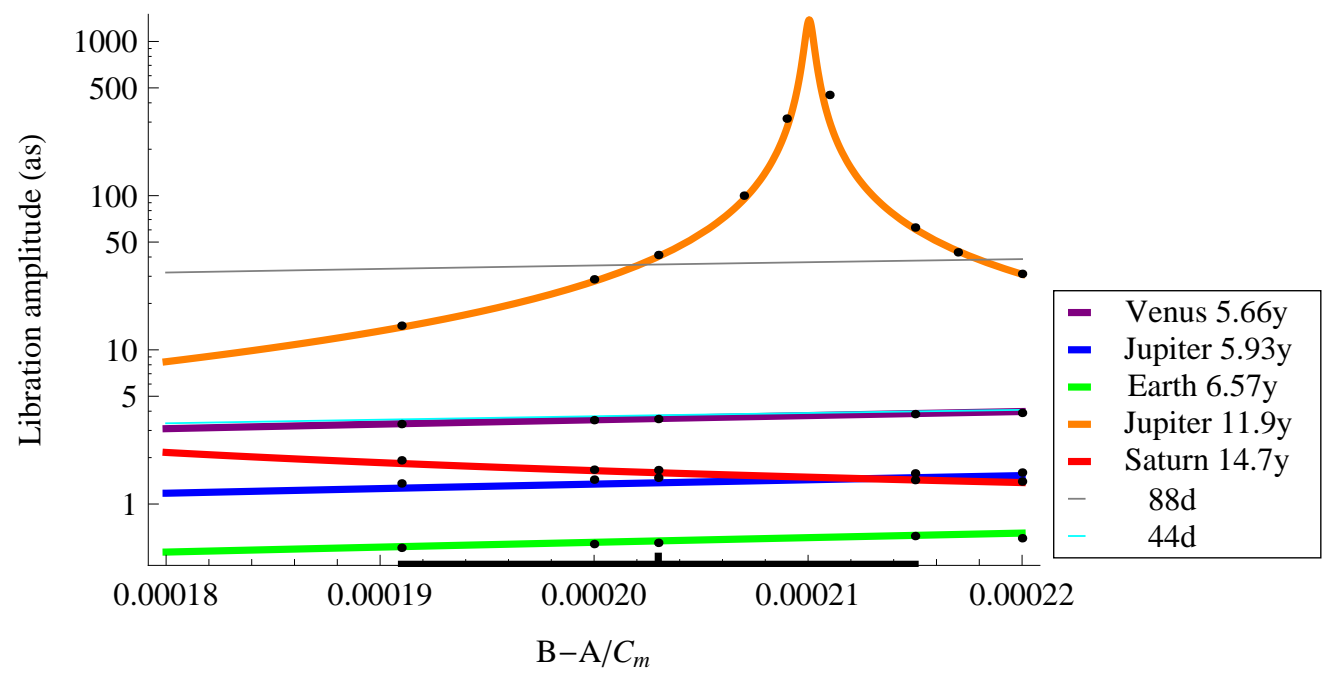

Figure 5: Amplitude (in arcsecond) of the forced librations $\psi_{m}(t)$ due to the planets as a function of the $(B-$ $A) / C_{m}$ ratio for Mercury. The nominal value for this ratio of $2.03 \times 10^{-4}$ is shown and the interval is the $1 \sigma$ uncertainty around this value from Margot et al (2007). This plot has been computed for an eccentricity value at J2000 epoch $(e=0.2056)$. The 88-day and 44-day physical libration amplitudes has also been plotted using thin lines. A logarithmic scale is used. The small dots are the results of the numerical integration.

with a good approximation, proportional to the moment of inertia ratio. These linear trends can be seen on Fig. 5 for most of the frequencies. For Venus, the amplitude goes from 3.31 as to 3.86 as in the $1 \sigma$ uncertainty interval around the $(B-A) / C_{m}$ ratio.

The small dots in Fig. 5 are obtained from a numerical integration of the equations of motion similar to the one performed in Peale et al (2009). They are very close to the values of our theoretical model. The fit is better here than in figure 6 of Peale et al (2009), because in this latter paper, the numerical amplitudes of $\psi_{11.86 y}$ were compared to the analytical solution $\gamma_{11.86 y}$.

The resonant effect with the $11.86 y$ planetary period is maximal if $(B-A) / C_{m}$ is equal to $2.1 \times 10^{-4}$. Since this ratio is between $1.91 \times 10^{-4}$ and $2.15 \times 10^{-4}(1 \sigma$ interval of Margot et al, 2007), the free libration period is likely between 11.7 and 12.5 years $(1 \sigma)$ and a large resonant effect with another planetary frequency (like Venus, the Earth, etc) is not possible. In order to have another planetary period equal to the free libration period, the $(B-A) / C_{m}$ ratio should be well outside the $3 \sigma$ interval, it should be at least three times larger than the present value. It has to be equal to $9.2 \times 10^{-4}, 8.4 \times 10^{-4}$ and $6.8 \times 10^{-4}$ respectively in order to be resonant with the $5.66 y$ (Venus), 5.93y (Jupiter) and 6.57y (Earth) planetary periods. For Saturn (14.73 y), this ratio should be much smaller $\left(1.36 \times 10^{-4}\right)$ than the measured value. Additionally, the $(B-A) / C_{m}$ ratio should be 20 time larger (again very far from the prediction) so that the libration amplitudes due to the other short-period terms (like the 1.1 year libration) are above the level of one arcsec.

The 88-day libration is also a function of the $(B-A) / C_{m}$ ratio, is plotted as a thin line in Fig. 5. Its amplitude is related to the moment of inertia by the following well-known equation (e.g. Peale 1972)

$$
A_{88 \mathrm{~d} \text { libration }}=\frac{3(B-A)}{2 C_{m}}\left(1-11 e^{2}+959 e^{4} / 48+\ldots\right)
$$

We see from Fig. 6 that the only libration phase affected by a change of moment of inertia value is the $11.86 \mathrm{y}$ libration. The phases for the other periods are almost constant, the variation is less than $0.1^{\circ}$. 


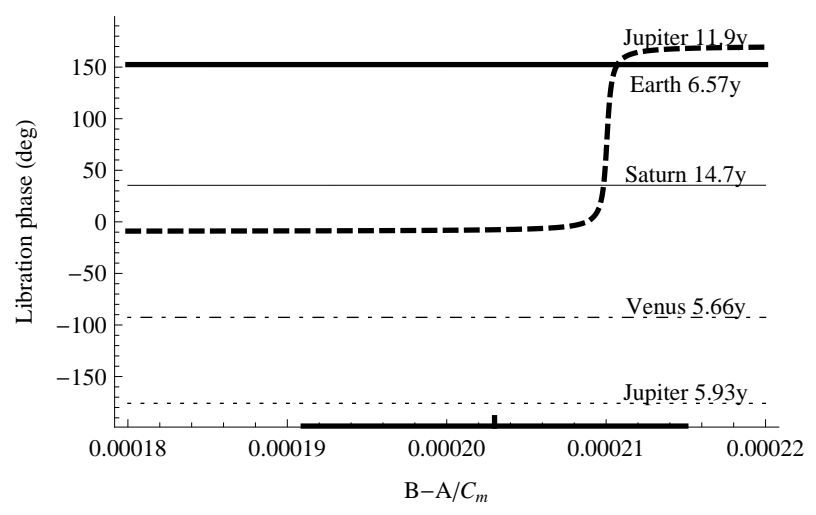

Figure 6: Phase (in degree) of the forced librations due to the planets as a function of the $(B-A) / C_{m}$ ratio.

\section{The model for the libration angle}

Over an interval of a few tens of years around J2000, the angle $\psi_{m}(t)$ can be modeled by the following formulation:

$$
\psi_{m}(t)=\psi_{0}+\left(1.5 n_{0}+\varpi_{t}\right) t+\sum_{i=1}^{\infty} A_{i} \sin \left(i n_{0} t\right)+\sum_{i=1}^{\infty} \psi_{i} \cos \left(w_{i} t+\phi_{\psi_{i}}\right),
$$

where $\varpi_{t}$ is the mean angular velocity of the longitude of the pericenter $\varpi(t)$. The first sum includes the forced libration with frequencies multiples of $2 \pi / 88$ days. This sum is infinite but practically, four waves are sufficient to explain $99.5 \%$ of the signal. A similar truncated sum has been proposed to improve the orientation model of Mercury (Margot, 2009). The amplitudes $A_{i}$ are given by a combination of two Kaula's eccentricity functions.

$$
A_{i}=\frac{1}{i^{2}} \frac{3(B-A)}{2 C_{m}}\left(G_{201-i}(e)-G_{201+i}(e)\right) .
$$

Eq. 22 follows from the expansion of Eq. 4 in terms of the mean anomaly.

The second sum includes the forced librations due to the planetary perturbations of the orbit, given in the previous sections. Again a very large number of terms is included in the summation, but practically using the five main terms instead of the full series gives an error smaller than 0.2 as for the rotation angle $\psi_{m}(t)$, and an error up to $0.6 \mathrm{as} / \mathrm{y}$ for the angular velocity $\psi_{m}^{\prime}(t)$.

Since the damping period of the free libration is short with respect of the age of the Solar System (Peale, 2005) and since no efficient excitation mechanism has been proposed up to now, the free libration is assumed to be damped in this study. Otherwise another term with frequency $w_{0}^{\prime}$ and with an undetermined amplitude and phase should be added to Eq. 21.

The libration rate $\psi_{m}^{\prime}(t)$ is obtained by taking the derivative of Eq. 21 with respect to time. Because of the time derivative and because each libration amplitude has to be multiplied by the frequency $w_{i}$, the short-period perturbations have a larger effect on the libration rate than on the libration angle. Therefore additional planetary perturbations of the orbit like the $1.11 \mathrm{y}$ perturbation have to be taken into account for highly precise rotation rate modeling. The libration rate values are given in Table 2 in the last column. The sum of all the short-period terms below the horizontal line in Table 2 goes up to $0.002 \mathrm{as} / \mathrm{d}$ for $\psi_{m}^{\prime}(t)$.

The residuals between the numerical integration and the sum of trigonometric function for the rotation angle $\psi_{m}(t)$ is less than 0.2 as. Since the original signal is of the order of $1 \mathrm{rad}$ or $2 \times 10^{5} \mathrm{as}$, this means that at least 6 digits are correct. For the temporal evolution of $\psi_{m}^{\prime}(t)$, the difference is less than $0.014 \mathrm{as} / \mathrm{d} . \psi_{m}^{\prime}(t)$ is close to $39 \mathrm{rad} / \mathrm{y}=2.2 \times 10^{4} \mathrm{as} / \mathrm{d}$. Again, at least 6 digits are correct. The radar measurement uncertainty on the instantaneous spin rate is of order one part per $10^{5}$ (uncertainty on $\psi_{m}^{\prime}(t)$ of about 0.14 as $/ \mathrm{d}$ for the X-band data to $1.3 \mathrm{as} / \mathrm{d}$ for the S-band data).

Figure 7 shows the time evolution of the planetary part of the libration angle $\psi_{m}(t)$ for different moment of inertia ratios. The lower panel shows the time evolution of the libration angular velocity.

A small variation in the moment of inertia ratio produces a large difference in the temporal evolution of the libration angle because of the resonance with the 11.86y libration. The difference between the 11.86y libration 

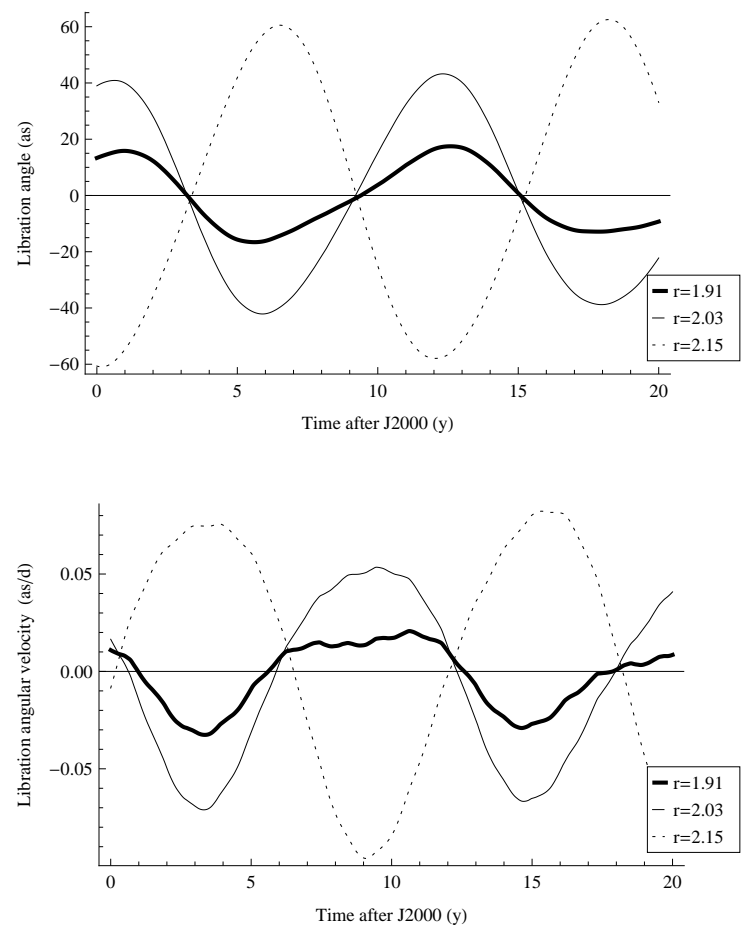

Figure 7: Temporal evolution of the planetary librations in the libration angle $\psi_{m}(t)$ (top) and of the libration angular velocity $\psi_{m}^{\prime}(t)$ (bottom) for different moment of inertia ratios $\left(r=10^{4}(B-A) / C_{m}\right)$. The librations with shorter period are more visible in the angular velocity than in the libration angle.

and the sum of the 5 main librations is small (a few arcseconds). The difference increases if the angular velocity is considered.

The 88-day forced libration and the librations due to the planetary perturbations of the orbit are comparable in amplitude (about 40 as) for a reasonable moment of inertia value. However the amplitudes of the angular velocity are completely different: about $3 \mathrm{as} / \mathrm{d}$ for the $88 \mathrm{~d}$ amplitude of the angular velocity and only 0.07 as $/ \mathrm{d}$ for the planetary amplitude. This is due to the large difference in the libration periods. If an orbiter measures angular displacements over a few Mercury orbits, the measurement will be very much affected by the long-period librations. If a radar antenna measures the spin rate, the measurement is largely insensitive to the (much smaller) effect of the long-period libration on spin rate.

\section{Fit of the moment of inertia ratio on the radar data}

Margot et al (2007) fitted their radar data with two models, both of which are consistent with the data. In their one-parameter model, they fit for the amplitude of the librations using a rotation model containing the 88-day libration and its harmonics. They find a best fit $(B-A) / C_{m}$ value of $2.04 \pm 0.06 \times 10^{-4}$ (1 $\sigma$ uncertainty). In their second model, they considered long-period librations but chose to be agnostic about their origin, allowing for the possibility of a free libration. This second model is fully dynamical and requires three parameters, but does not include the planetary perturbations of the orbit investigated here. Their second model yields a $(B-A) / C_{m}$ value of $2.03 \pm 0.04 \times 10^{-4}$. Here we use the full model with the librations due to the planetary perturbations of the orbit (see previous section) in order to find the best fit moment of inertia ratio and its uncertainty. This model has only one free parameter. The moment of inertia ratio control the amplitudes of the 88d libration and its multiples, the amplitudes of the long-period forced librations and their phases. We do not consider any free libration in our model. The radar data cover a time span of about 4.5 years after the calendar year 2002 .

We use the numerical solution of the differential equation for the motion of the mantle. Figure 8 shows the radar data and their uncertainties superposed with the solution of the numerical integration of $\psi_{m}^{\prime}(t)$ for different moment of inertia ratios. 


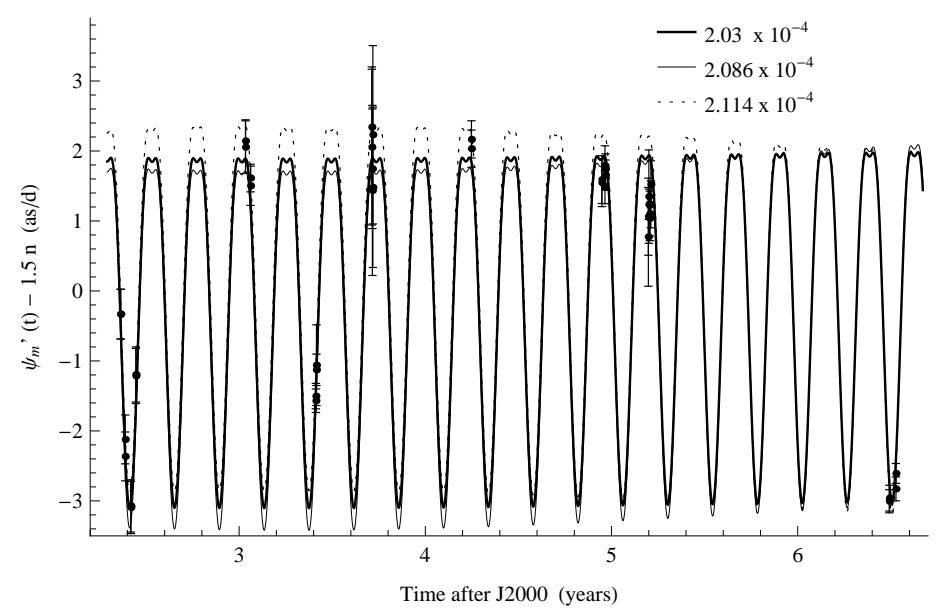

Figure 8: Temporal evolution of the libration angular velocity $\psi_{m}^{\prime}(t)-1.5 n$ for different moment of inertia ratios. This is the solution of the numerical integration. The radar data and their uncertainties are also plotted.

The uncertainties on the measurements are at the same level as the difference between the solution for different moment of inertia values, indicating that it is currently difficult to use long-period librations to discriminate between $(B-A) / C_{m}$ values.

The goodness of the fit is estimated by computing the reduced $\chi^{2}$, which is the sum of the squared difference between the model and the data, weighted by the data uncertainty, and divided by the number of degrees of freedom.

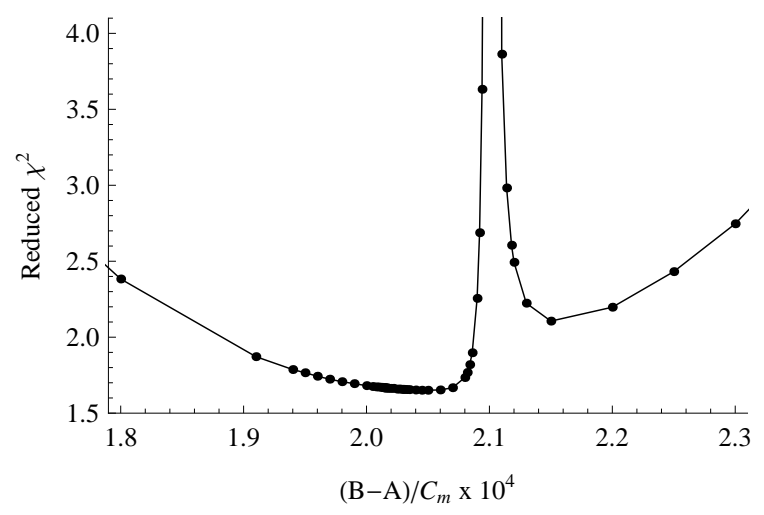

Figure 9: $\chi^{2}$ for different moment of inertia ratios.

Figure 9 shows the reduced $\chi^{2}$ for different moment of inertia ratios. The best fit value is $(B-A) / C_{m}=$ $2.06 \pm 0.09 \times 10^{-4}$ where the limits are given for a $95 \%$ confidence interval $(2 \sigma)$. Close to the minimum, the curve is flat, meaning that the ratio is not very well constrained.

The analytical model can also be used to compare the temporal variations of the angular velocity to the radar data. But since the numerical solution is very close to the analytical model, results are statistically consistent. The advantage is that partial derivatives of the angular velocity can be easily computed with respect to the moment of inertia ratio. The best fit value for $(B-A) / C_{m}$ is $2.05 \pm 0.10 \times 10^{-4}$. This moment of inertia value corresponds to an amplitude for the 11.86y libration of 57 as and a 11.86y libration rate of 0.08 as $/$ d. The best fit value of $(B-A) / C_{m}=2.03 \times 10^{-4}$ of Margot et al (2007) corresponds to $\psi_{11.86 y}$ of about 40 as.

Our best fit value for $(B-A) / C_{m}$ with the full libration model is not statistically different from the best fit value with only the 88 -day librations and its multiples. The $1 \sigma$ uncertainty is also similar. Both models accommodate the data. The fit of the radar data is not affected much by the planetary librations because the long-period forced libration amplitude in the spin rate (around 0.05 as/d) is smaller than the present radar data 
uncertainty (about 0.15 as/d). Should the moment of inertia ratio be such that a long-period forced libration is detectable in the radar data, our model could explain both the long- and short-period forced librations with only one unknown parameter.

The question of the presence of an additional long-period libration (the free libration) with an arbitrary amplitude and phase is still open. In the presence of a free libration, a complete model would still require three free parameters.

\section{Discussion}

The amplitudes of $\psi_{i}$ given by the theoretical equation (Eq. 17) match very well the values given by the numerical integration of the system, the difference is less than $1 \%$ (see the dots in Fig. 5). This validates the harmonic oscillator model and confirms the validity of our simplifications. Additionally, the mantle motion has been integrated numerically without any variations in $a(t)$ and $e(t)$ and the change for the libration is less than 0.2 as. It confirms our hypothesis that the time variations in $a(t)$ and $e(t)$ may be neglected for computing the long-period librations.

All the amplitudes are given around the J2000 epoch. Since the orbital elements are changing with time, the amplitudes will be different for another epoch. The free libration period is not constant with time since $e(t)$ and $n(t)$ are changing quantities.

The theoretical amplitudes are also very close to the results of Peale et al (2009) and Dufey et al (2009). The phases $\phi_{i}$ obtained with a numerical integration of equation (Eq. 1) are also very close to the theoretical value. A limitation is when the $w_{i}$ frequency is too close to the resonant frequency, i.e. $\left|w_{0}-w_{i}\right|$ is smaller than $0.01 \mathrm{rad} / \mathrm{y}$. The amplitude $\gamma_{11.86 y}$ is larger than 50 as and the two solutions differ. In that case, the numerical determination of the $w_{0}$ and $w_{i}$ amplitudes is not perfect because their periods are too close and because the free libration cannot be fully damped numerically. Additionally, the harmonic oscillator model may not be accurate so close to the resonance.

Another limitating factor of the model is the frequency range: if the frequency is large (i.e. larger than $20 \mathrm{rad} / \mathrm{y}$ ), the model may not be exact since the equation of motion of the mantle is averaged over one orbital period.

The amplitudes of the librations are very sensitive to the choice of the exact value for the perturbating frequency. A small change in the $w_{i}$ value (for example using another algorithm for frequency determination on the orbital element) may lead to differences in the libration amplitude.

The rotation angle $\psi_{m}(t)$ and angle $\gamma_{m}(t)$ are not equal and their periodic amplitudes also differ. The difference is approximatively the $\lambda(t)$ factor. Even their phase may be different (up to $180^{\circ}$ ). The quantity commonly measured or extracted from numerical integration of the equations of motion is the rotation angle $\psi_{m}(t)$ while $\gamma_{m}(t)$ is more convenient for computing the torque.

\section{Summary}

The solar torque on Mercury leads to rotation variations with a main signal at the orbital period of 88 days. The planetary perturbations induce variations in the orbital elements of Mercury. This changes the averaged solar torque on Mercury's axial asymmetry and leads to long-period forced librations in longitude. Usually, only five waves need to be considered: Jupiter (11.86y and 5.93y), Venus (5.66y), the Earth (6.57y) and Saturn (14.73y). For more precise studies or when rotation data precision will be below the arcsecond level, more waves will be needed.

Mercury's libration in longitude due to planetary perturbations of Mercury's orbit can be predicted with a simple analytical model based on the damped harmonic oscillator. If the planetary perturbations on the $1.5 M(t)+\varpi(t)$ variable are known from an orbital theory for each frequency, Eq. 17 and Eq. 19 give the amplitude and phase of the induced librations. The proximity of the five planetary periods to the free libration period of about 12 years, explains why these perturbations induce libration amplitude above 0.5 arcsecond. In particular, the free libration period can be very close to the Jupiter forcing period with a period of 11.86 year and can result in a very large libration amplitude at that period. Far from the resonant frequency, the libration amplitudes will be well below the arcsecond level even if the orbit perturbation is large.

These long-period forced librations are independent of the excitation state of the free libration. If, for any 
unknown reason, Mercury experiences some free libration, its amplitude and phase will be arbitrary while the amplitude and phases of the forced librations are given by the theoretical equation.

Since the free libration frequency is proportional to the square root of $(B-A) / C_{m}$, the link between the libration amplitude and the moment of inertia ratio is direct (see Eq. 18). The 11.86y libration amplitude shows a resonant effect with the $(B-A) / C_{m}$ value while the other four planetary libration amplitudes show a small linear trend with respect to the $(B-A) / C_{m}$ ratio.

The fit of the model on the radar data gives a $(B-A) / C_{m}$ value of $2.05 \pm 0.10 \times 10^{-4}$. This value does not differ statistically from the value of Margot et al (2007) because the current uncertainties on the radar measurements of the angular velocity $\psi_{m}^{\prime}(t)$ are about 3 time larger than the contribution to the spin rate signature from the 11.86 y libration due to the planetary perturbation for $(B-A) / C_{m}$ around $2 \times 10^{-4}$. Therefore without any dramatic improvement in the radar precision in the coming years and without data covering a longer time interval (longer than 12 years), the separation between the different librations may not be possible in the near future if one fits the libration amplitudes on the radar data. Nevertheless, we have demonstrated the relation between those amplitudes and the only unknown parameter influencing all the amplitudes, the same moment of inertia ratio $(B-A) / C_{m}$. Since the phase of the planetary librations are given by the theory based on celestial mechanics and Mercury's orbit, a global fit of this ratio on the libration data is still possible, even if the different waves may not be separable. The determination of this ratio coupled with a better determination of the second order coefficients of the gravity field will dramatically improve our knowledge of the interior structure of Mercury. Additionally, a complementary view of the rotation of Mercury will be given by spacecraft measurement with the MESSENGER and BepiColombo mission since they will measure the rotation angle and not the angular velocity, the rotation angle being much more sensitive to the long-period librations.

Because the periods of the librations are different, the long-period forced librations do not change the amplitude of the 88-day forced libration. Anyway even if these five main long-period librations cannot be separated presently into the data, their amplitudes and phases need to be included in the rotation model.

\section{Acknowledgments}

We thank Tim Van Hoolst and Véronique Dehant for helpful comments and discussion. M.Y. was financially supported by the Belgian PRODEX program managed by the European Space Agency in collaboration with the Belgian Federal Science Policy Office. J.L.M. is supported in part by NASA grants NNX08AO24G/NNX09AQ69G from the Planetary Astronomy Program and NNX07AV78G/NNX09AR45G from the MESSENGER mission. S.J.P. is supported in part by NASA grants NNG05GK58G from the Planetary Geology and Geophysics Program and NNG06GF42G from the Origins of Solar Systems Program and by the MESSENGER mission under contract DTM-3250-09. M.Y. was financially supported by the Belgian PRODEX program managed by the European Space Agency in collaboration with the Belgian Federal Science Policy Office.

\section{References}

- Dufey, J., Lemaitre, A., Rambaux, N., 2008. Planetary perturbations on Mercurys libration in longitude. Cel. Mech. Dyn. Astr. 101, 141157.

- Dufey, J., Noyelles, B., Rambaux, N., Lemaitre, A., 2009. Latitudinal librations of Mercury with a fluid core. Icarus 203, 1-12.

- Kaula, W., 1966. Theory of Satellite Geodesy, Blaisdell Publishing Company, Waltham, MA.

- Margot, J. L., Peale, S.J., Jurgens, R.F., Slade, M.A., Holin, I.V., 2007. Large longitude libration of Mercury reveals a molten core. Science 316, 710-715.

- Margot, J.-L., 2009, A Mercury orientation model including non-zero obliquity and librations. Cel. Mech. Dyn. Astr., 105, 329-336.

- Murray, C.D., and Dermott S.F., Solar System Dynamics, Cambridge University Press, 1999.

- Peale, S.J., 1972, Determination of parameters related to the interior of Mercury, Icarus 17, 168-173.

- Peale, S.J., 2005. The free precession and libration of Mercury. Icarus 178, 4-18. 
- Peale, S.J., Yseboodt M., Margot J.L., 2007. Long-period forcing of Mercury's libration in longitude. Icarus 187, 365-373.

- Peale, S.J., Margot, J.L., Yseboodt M., 2009. Resonant forcing of Mercury's libration in longitude. Icarus 199, 1-8. 